\title{
ВЛИЯНИЕ ЭМОЦИОНАЛЬНОГО СОСТОЯНИЯ И ДИСПОЗИЦИОНАЛЬНОЙ РАДОСТИ НА СКОРОСТЬ РАСПОЗНАВАНИЯ ЭМОЦИЙ ПО ВЫРАЖЕНИЮ ЛИЦА
}

\author{
В.В. ОВСЯННИКОВА
}

${ }^{a}$ Национальный исследовательский университет «Высшая школа экономики», 101000, Россия, Москва, ул. Мясницкая, д. 20

\section{Резюме}

В предыдущих работах автора было показано, что может быть получен эффект конгруэнтности настроения или эффект конгруэнтности черты. В настоящем исследовании изучалось влияние эмоционального состояния и диспозициональной радости на эффективность распознавания эмоций по выражению лица. Проведено экспериментальное исследование с участием двух групп испытуемых. Общую выборку составили 39 человек. Эмоциональное состояние участников измерялось с помощью самоотчетной методики (ШПАНА). Воздействие на текущее настроение проводилось с помощью процедуры индукции эмоций, которая включала показ испытуемому видеоролика с «радостной» или «нейтральной» эмоциональной окраской. Для измерения скорости переработки эмоциональной информации использовалась компьютерная методика, в которой участник выполнял задание на распознавание эмоций по выражению лица. В работе проверялась гипотеза о наличии эффекта конгруэнтности при переработке положительно окрашенной информации. Предполагалось, что положительное эмоциональное состояние и диспозициональная радость повышают скорость переработки положительно окрашенного материала и не оказывают влияния на переработку стимулов с негативной эмоциональной окраской. Проверка эффективности процедуры индукции эмоций показала, что она оказалась частично успешной. Был получен эффект конгруэнтности для диспозициональной радости: обнаружены связи высокой выраженности этой черты с повышением скорости распознавания эмоции радости на изображениях лиц. Влияние положительного эмоционального состояния заключалось в уменьшении скорости распознавания экспрессии радости. Результаты показали, что эффект конгруэнтности проявляется по-разному для черты по сравнению с эмоциональным состоянием. В целом проведенное исследование дает информацию о механизмах распознавания эмоций.

Ключевые слова: распознавание эмоций, эмоциональное состояние, индукция эмоций, конгруэнтность.

Данное научное исследование (№ 14-01-0171) выполнено при поддержке Программы «Научный фонд НИУ ВШЭ» в 2014-2015 гг. 
Значительное число современных исследований в области изучения эмоций посвящено вопросу о том, каким образом происходит переработка эмоционально окрашенного материала. Преимущественно они руководствуются представлениями об эмоциональной конгруэнтности, согласно которым люди более эффективно воспринимают, запоминают, обращают внимание на тот материал, эмоциональная окраска которого соответствует их текущему эмоциональному состоянию. Так, находясь в радостном настроении, человек будет лучше запоминать позитивные аспекты ситуации, интерпретировать события в положительном ключе, в то время как тот, кто грустит, будет придавать своим оценкам негативную окраску.

Возникновение эффекта конгруэнтности с позиции модели ассоциативной сети обосновал Г. Бауэр (Bower, 1981), описывая эффекты влияния эмоций на память. Он предположил, что в семантической сети есть эмоциональные узлы, активация которых усиливается в момент переживания человеком соответствующих эмоций. Это делает более доступной для переработки всю информацию, связанную с этими узлами (например, воспоминание о каком-то событии). Эффекты конгруэнтности настроения были получены не только на примере памяти, но для процессов вынесения суждений, внимания, восприятия и др.

Идея конгруэнтности касается не только роли состояний и настроений в переработке информации, но позже стала распространяться также на устойчивые эмоциональные характеристики - личностные черты. В этом случае индивидуальные различия в переработке эмоциональной информации объясняются тем, что люди склонны реагировать способом, конгруэнтным содержанию черты. Так, у человека, который имеет высокую выраженность какой-либо негативно окрашенной черты (например, агрессивности), преимущество в переработке будет получать негативно окрашенный материал.

Исследователи отмечают, что, помимо изучения независимо друг от друга роли эмоциональных состояний и черт в переработке эмоциональных стимулов, важно принимать в расчет их совместное влияние. Сочетания тех или иных личностных характеристик и эмоциональных состояний могут способствовать или препятствовать возникновению эффектов конгруэнтности. Ш. Растинг предлагает два подхода к анализу взаимосвязей этих переменных, которые описывают возникновение эффектов конгруэнтности. В рамках первого подхода предполагается, что если у человека выражены те или иные устойчивые эмоциональные черты, то он предрасположен переживать определенные эмоции и настроения, которые, в свою очередь, оказывают влияние на переработку эмоциональной информации. Согласно другому подходу, эффекты конгруэнтности текущего состояния и эмоциональной окраски материала зависят от индивидуальных различий в личностных чертах (Rusting, 1998).

В поиске эффектов совместного влияния устойчивых и изменчивых эмоциональных характеристик на переработку эмоциональной информации проведено большое количество исследований (Tamir et al., 2002; 
Rafienia et al., 2008; Edwards, 2014). Их результаты зачастую мало согласуются между собой, с трудом поддаются обобщению как по причине разнообразия методик, которые авторы используют для измерения эмоциональных характеристик и особенностей переработки, так и изза того, что искомые эффекты слабо выражены и неустойчивы. В качестве примера одного из недавних исследований, в котором получены данные о совместном влиянии состояний и черт, можно привести исследование Т. Кварто с соавт. (Quarto et al., 2014). В этой работе изучалось влияние тревожности и различных эмоциональных состояний на имплицитную переработку экспрессии. Задача испытуемых состояла в том, чтобы определить пол человека по изображению лица. При этом предъявляемые лица выражали различные эмоции (радость, гнев, нейтральное выражение). Выполнение этого задания имело акустическое сопровождение: участники слушали либо успокаивающие «терапевтические» звуки, либо нервирующий шум, либо посторонние звуки отсутствовали.

Оказалось, что испытуемые быстрее реагировали на лица с экспрессией радости, прослушивая успокаивающие звуки, по сравнению со временем их реакции на лица с экспрессией гнева при прослушивании шума. Авторы объясняют этот результат тем, что вызванное прослушиванием шума негативное эмоциональное состояние влияло на имплицитную переработку выражений лиц, приводя к переключению внимания в сторону «злых» лиц, что выражалось в замедлении времени реакции при определении пола. Наоборот, переработка лиц, выражающих радость, при прослушивании успокаивающей музыки требовала меньше когнитивных ресурсов.

Также результаты показали наличие совместного влияния состояний и черт. По сравнению с условием отсутствия звукового сопровождения высоко тревожные испытуемые реагировали быстрее при прослушивании успокаивающей музыки, а у испытуемых с низкой тревожностью время реакции было больше при прослушивании раздражающего шума. Авторы интерпретируют этот результат как различия людей с высокой и низкой личностной тревожностью в отношении чувствительности к эффектам индукции настроения. Высокотревожные испытуемые лучше поддаются успокаивающему воздействию приятных звуков по сравнению с низкотревожными испытуемыми. В то же время люди с низкой тревожностью сильнее подвержены влиянию раздражающих звуков. Таким образом, исследование показало, что на переработку эмоциональной информации влияют как состояния, так и эмоциональные черты.

В настоящем исследовании предпринята попытка изучить совместное влияние двух эмоциональных характеристик на эффективность распознавания эмоций по выражению лица. Проведен эксперимент с участием двух групп испытуемых, эмоциональное состояние которых изменялось с помощью процедуры индукции эмоций. У одной группы вызывалось положительное эмоциональное состояние, у другой группы поддерживалось нейтральное состояние. В качестве 
стабильной личностной характеристики измерялась диспозициональная радость. В исследовании проверялась гипотеза о том, что положительное эмоциональное состояние и диспозициональная радость повышают скорость переработки положительно окрашенного материала и не оказывают влияния на переработку стимулов с негативной эмоциональной окраской.

\section{Методика}

\section{Испьтутемье}

В исследовании приняли участие 39 испытуемых (из них 82\% женского пола) в возрасте от 17 до 22 лет $(\mathrm{M}=18.6, \mathrm{SD}=1.0)$, студенты гуманитарного вуза.

\section{Материаль}

Скорость переработки эмоционально окрашенной информации измерялась с помощью специально разработанной компьютерной методики, в качестве стимулов использовались изображения лица, выражающие четыре категории эмоций (гнев, радость, страх, печаль) и нейтральное состояние.

Изображения лиц были выбраны из базы ADFES (Van der Schalk et al., 2011). В данном исследовании использовался набор эмоциональных лиц, который применялся ранее в работе Ю.А. Кожуховой (Кожухова, 2016). Каждая эмоциональная категория представлена четырьмя изображениями мужского и женского лица, выражающими соответствующую эмоцию. Также было отобрано по четыре мужских и женских лица, не выражающих эмоций (нейтраль- ное выражение). Таким образом, использовалось восемь эмоциональных и восемь нейтральных изображений лиц.

Для предъявления стимулов, инструкций и регистрации ответов участников использовалась программа OGAMA v. 5.0. Изображения размером 14 см на 21 см, представленные в градациях серого цвета, предъявлялись в центре экрана на мониторе с диагональю 15”.

Данное исследование включало процедуру индукции эмоционального состояния, которая состоит в показе испытуемому короткого видеоролика с положительной или нейтральной эмоциональной окраской. Этот метод широко применяется в современных исследованиях для индукции эмоций в лабораторных условиях (Westermann et al., 1996). Испытуемым экспериментальной группы для индукции положительных эмоций показывали «веселый» мультфильм, в котором по сюжету два осьминога попадают в различные забавные ситуации («Oktapodi», продолжительность - 2 мин 25 сек). Участники контрольной группы смотрели «нейтральный» видеоролик, в котором показаны движущиеся в различных направлениях цветные линии («Палочки», продолжительность - 2 мин 50 сек). Эффективность индукции эмоций с помощью данного видеоматериала была проверена в рамках пилотажного этапа кросс-культурного исследования Ю.Е. Ченцовой-Даттон и Д.В. Люсина. Результаты показали, что после просмотра видеороликов состояние испытуемых изменялось согласно их эмоциональной окраске (Chentsova Dutton et al., 2013). 
Для измерения текущего эмоционального состояния использовалась методика ШПАНА (русскоязычная адаптация методики PANAS; Осин, 2012). Методика состоит из 20 прилагательных, посредством которых испытуемый должен оценить свое текущее состояние, дав балльную оценку по шкале от 1 (почти или совсем нет) до 5 (очень сильно). Методика позволяет оценить эмоциональное состояние по двум шкалам - Позитивный аффект и Негативный аффект.

Для измерения диспозициональной радости использовались русские адаптации двух методик - Шкалы субъективного счастья С. Любомирски и Шкалы удовлетворенности жизнью Э. Динера (Осин, Леонтьев, 2008). Шкала субъективного счастья состоит из четырех утверждений, касающихся радости и счастья в жизни, каждое из которых нужно оценить по шкале от 1 до 7. Шкала удовлетворенности жизнью содержит пять утверждений про восприятие своей жизни, по каждому из которых нужно выразить степень согласия по шкале от 1 до 7. Баллы по шкалам удовлетворенности жизнью и субъективного счастья суммировались. Полученный итоговый результат рассматривался как показатель диспозициональной радости.

\section{Прочедура}

Процедура исследования выполнялась в несколько этапов. На первом этапе участник заполнял опросник ШПАНА для оценки своего текущего эмоционального состояния перед началом исследования. После этого он проходил тренировочную серию компьютерной методики, измеряющую время реакции при распознавании эмоций по выражению лица. Она включала пять проб, после каждой из которых испытуемым сообщалось, насколько быстро и правильно они ответили. Стимулы из тренировочных серий не использовались в основных.

На следующем этапе испытуемый переходил к просмотру одного из двух видеороликов - «веселого» или «нейтрального» в зависимости от принадлежности к экспериментальной или контрольной группе. С помощью «веселого» ролика индуцировалось положительное эмоциональное состояние (экспериментальная группа); посредством «нейтрального» ролика поддерживалось условно нейтральное состояние (контрольная группа).

Сразу после просмотра видеоролика участник выполнял основную серию компьютерной методики на измерение времени реакции. Его задача заключалась в том, чтобы как можно быстрее и точнее ответить, нажав на заданную клавишу, выражает ли лицо на фотографии какиелибо эмоции. О появлении изображения лица предупреждал фиксационный крест (1.5 сек), после изображения лица на 2 сек появлялось маскирующее изображение. Все 16 фотографий лиц предъявлялись в фиксированной последовательности два раза (всего 32 пробы).

На последнем этапе испытуемый повторно оценивал свое эмоциональное состояние с помощью методики ШПАНА. Кроме этого, он заполнял опросники на эмоциональные лич- 
ностные черты (в данной работе анализируется только диспозициональная радость).

Работа с каждым испытуемым проводилась индивидуально, полная процедура занимала в среднем 40 мин.

\section{Результаты}

Из обработки результатов методики распознавания эмоций по выражению лица были исключены пробы, в которых испытуемые ответили неверно (5\% всех проб). Также исключались пробы, в которых время реакции выходило за рамки трех стандартных отклонений от среднего времени реакции ответов каждого участника (3\% всех проб).

Для каждого испытуемого был посчитан показатель времени реакции - среднее арифметическое времени реакции для каждого типа эмоционально окрашенных лиц, для нейтральных лиц, а также для всех негативно окрашенных лиц (выражающих гнев, страх, печаль).

В первую очередь была проведена оценка эффективности процедуры индукции положительного эмоционального состояния у испытуемых экспериментальной группы. В случае успешной индукции оценки Позитивного аффекта во втором замере (после выполнения методики распознавания эмоций по выражению лица) должны быть выше, чем оценки по этой шкале в первом замере (до выполнения методики); значения Негативного аффекта, наоборот, должны снизиться или остаться без изменения. Описательные статистики оценок эмоционального состояния в двух замерах у участников двух групп представлены в таблице 1.

Таблица показывает, что у группы с индукцией положительных эмоций оценка Позитивного аффекта незначительно возросла от первого измерения ко второму, а оценка Негативного аффекта снизилась. У «нейтральной» группы значения по обеим шкалам снизились. Критерий Вилкоксона показал, что различия в средних значениях являются статистически значимыми только у испытуемых экспериментальной группы по шкале Негативного аффекта $(\mathrm{Z}=-2.305$

Таблица 1

Описательные статистики двух измерений эмоционального состояния у контрольной и экспериментальной групп

\begin{tabular}{|c|c|c|c|c|}
\hline $\begin{array}{c}\text { Измерение } \\
\text { эмоционального } \\
\text { состояния }\end{array}$ & \multicolumn{2}{|c|}{$\begin{array}{c}\text { Группа с индукцией } \\
\text { положительного } \\
\text { эмоционального состояния }\end{array}$} & \multicolumn{2}{|c|}{$\begin{array}{c}\text { Группа с поддержанием } \\
\text { нейтрального эмоционального } \\
\text { состояния (контрольная группа) }\end{array}$} \\
\cline { 2 - 5 } & $\begin{array}{c}\text { Позитивный } \\
\text { аффект }\end{array}$ & $\begin{array}{c}\text { Негативный } \\
\text { аффект }\end{array}$ & $\begin{array}{c}\text { Позитивный } \\
\text { аффект }\end{array}$ & $\begin{array}{c}\text { Негативный } \\
\text { аффект }\end{array}$ \\
\hline № 1 & $31.40(5.13)$ & $12.70(2.83)$ & $31.47(6.03)$ & $13.00(2.89)$ \\
\hline$№ 2$ & $31.65(8.21)$ & $12.00(2.41)$ & $31.26(6.67)$ & $11.68(2.16)$ \\
\hline
\end{tabular}

Примечание. В ячейках таблицы указаны средние арифметические значения, в скобках приведены стандартные отклонения. 
при $p=0.021)$. Таким образом, в силу того, что оценки Негативного аффекта в этой группе значимо снизились, а оценка Позитивного аффекта возросла (хотя различия и не достигли уровня значимости), индукцию положительного эмоционального состояния можно рассматривать как частично успешную.

Для проверки гипотезы о влиянии диспозициональной радости и эмоционального состояния на скорость распознавания эмоций был проведен двухфакторный дисперсионный анализ $2 \times 2$, в котором зависимой переменной выступало время реакции при распознавании радости по выражению лица, а фиксированными факторами являлись тип эмоционального состояния (положительное/нейтральное) и выраженность диспозициональной радости (высокая/низкая).

Нужно отметить, что общая выборка испытуемых была разделена на две группы с высокой и низкой диспозициональной радостью на основании медианного критерия. Значения выше медианы составили группу с высокой выраженностью диспозициональной радости, значения ниже медианы - группу с низкой выраженностью диспозициональной радости. Описательные статистики значений времени реакции в сравниваемых подгруппах представлены в таблице 2.

Таблица показывает, что если принимать во внимание отдельно влияние фактора эмоционального состояния, то время реакции распознавания эмоции радости по выражению лица у испытуемых в положительном эмоциональном состоянии больше, чем время реакции у участников в нейтральном состоянии (830.63 и 734.32 соответственно). Основной эффект фактора текущего эмоционального состояния является статистически значимым: $\mathrm{F}(1,32)=$ 4.89, $\eta^{2}=0.0036, p=0.034$.

Средние значения времени реакции распознавания радости у группы с высокой выраженностью диспозициональной радости ниже по сравнению со временем реакции у группы с низким уровнем этой черты (735.92 и 829.03 соответственно). Основной

Таблица 2

Описательные статистики значений времени реакции при распознавании радости в подгруппах испытуемых с высокой и низкой диспозициональной радостью и разным типом эмоционального состояния

\begin{tabular}{|c|c|c|c|c|c|}
\hline & \multicolumn{4}{|c|}{ Диспозициональная радость } \\
\hline & & \multicolumn{2}{|c|}{ Высокий уровень } & \multicolumn{2}{|c|}{ Низкий уровень } \\
\hline & & $\mathrm{M}(\mathrm{SD})$ & $\mathrm{N}$ & $\mathrm{M}(\mathrm{SD})$ & $\mathrm{N}$ \\
\hline \multirow{3}{*}{ 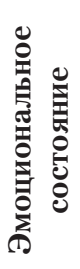 } & $\begin{array}{l}\text { Положительное } \\
\text { эмоциональное состояние }\end{array}$ & $\begin{array}{c}767.70 \\
(116.50)\end{array}$ & 10 & $\begin{array}{c}893.56 \\
(161.60)\end{array}$ & 9 \\
\hline & $\begin{array}{l}\text { Нейтральное } \\
\text { эмоциональное состояние }\end{array}$ & $\begin{array}{c}704.14 \\
(123.37)\end{array}$ & 7 & $\begin{array}{c}764.50 \\
(111.58)\end{array}$ & 10 \\
\hline & $\begin{array}{l}\text { Общее среднее арифм. } \\
\text { (стандартное отклонение) }\end{array}$ & $\begin{array}{c}735.92 \\
(119.94)\end{array}$ & 17 & $\begin{array}{c}829.03 \\
(136.60)\end{array}$ & 19 \\
\hline
\end{tabular}


эффект фактора диспозициональной радости также является статистически значимым: $\mathrm{F}(1,32)=4.57, \eta^{2}=0.0033$, $p=0.040$.

Взаимодействие факторов состояния и черты не достигло уровня статистической значимости $(p>0.05)$.

Чтобы проверить, что искомые эффекты положительного эмоционального состояния и диспозициональная радость характеризуют именно переработку «радостных» выражений лица и не специфичны для переработки негативно окрашенных стимулов, был проведен двухфакторный дисперсионный анализ $2 \times 2$, в котором зависимой переменной выступало время реакции при распознавании негативных эмоций по выражению лица. Это время реакции вычислялось для каждого испытуемого как среднее арифметическое значений времени реакции при восприятии лиц, выражающих гнев, страх и печаль. Межгрупповые факторы остались те же - тип эмоционального состояния (положительное/нейтральное) и выраженность диспозициональной радости (высокая/низкая). Описательные стати- стики значений времени реакции в сравниваемых подгруппах представлены в таблице 3.

Таблица 3 показывает, что среднее время реакции распознавания негативных эмоций у испытуемых в положительном эмоциональном состоянии равно 886.79, а у испытуемых в нейтральном состоянии оно меньше и составляет 788.90. У группы с низким уровнем диспозициональной радости среднее время реакции больше, чем у группы с высоким уровнем этой характеристики, - 891.11 и 784.57 соответственно. В данном случае не получено статистически значимых основных эффектов факторов текущего эмоционального состояния и черты, а также их взаимодействия $(p>0.05)$.

\section{Обсуждение результатов}

В работе проверялась гипотеза о том, что положительное эмоциональное состояние и диспозициональная радость повышают скорость переработки положительно окрашенного материала (в данном случае изображений лица) и не оказывают влияния

Таблица 3

Описательные статистики значений времени реакции при распознавании негативных эмоций в подгруппах испытуемых с высокой и низкой диспозициональной радостью и разным типом эмоционального состояния

\begin{tabular}{|c|c|c|c|c|c|}
\hline & \multicolumn{4}{|c|}{ Диспозициональная радость } \\
\hline & & \multicolumn{2}{|c|}{ Высокий уровень } & \multicolumn{2}{|c|}{ Низкий уровень } \\
\hline & & $\mathrm{M}(\mathrm{SD})$ & $\mathrm{N}$ & $\mathrm{M}(\mathrm{SD})$ & $\mathrm{N}$ \\
\hline \multirow{2}{*}{ 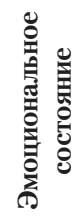 } & \begin{tabular}{|l} 
Положительное \\
эмоциональное состояние
\end{tabular} & $\begin{array}{c}840.85 \\
(136.65)\end{array}$ & 10 & $\begin{array}{c}932.72 \\
(223.26)\end{array}$ & 9 \\
\hline & $\begin{array}{l}\text { Нейтральное } \\
\text { эмоциональное состояние }\end{array}$ & $\begin{array}{c}728.29 \\
(105.76)\end{array}$ & 7 & $\begin{array}{c}849.50 \\
(215.45)\end{array}$ & 10 \\
\hline
\end{tabular}


на переработку стимулов с негативной эмоциональной окраской.

В проведенном исследовании было показано наличие влияния на переработку эмоционально окрашенного материала обеих изучаемых характеристик - эмоционального состояния и диспозициональной радости. Однако высокая выраженность диспозициональной радости по сравнению с ее низким уровнем сопровождалась ускорением распознавания эмоций на «радостных» лицах, в то время как положительное эмоциональное состояние привело к замедлению скорости их распознавания. Полученные данные соответствуют «чистому» эффекту конгруэнтности между выраженностью диспозициональной радости и временем переработки положительно окрашенной информации, при котором переработка радости выполнялась быстрее тогда, когда испытуемые переживали положительные эмоции. В случае эмоционального состояния эффект конгруэнтности оказал противоположное действие, увеличивалось время ответа, влияние на восприятие экспрессии радости. Нужно отметить, что если не учитывать текущее эмоциональное состояние, то этот результат противоречит полученному в других работах, в которых показано, что происходит ускорение переработки положительно окрашенных стимулов (например: Calvo et al., 2010).

Как уже отмечалось во вводной части, не во всех представленных в литературе исследованиях удается получить результаты, характеризующие как эффекты конгруэнтности для отдельных черт или состояний в переработке информации, так и взаимодействие изучаемых эмоциональных характеристик. Зачастую авторы объясняют это возможным влиянием других факторов на искомые эффекты. В качестве таковых рассматриваются, например, различия в форматах ответов в заданиях на распознавание эмоций (Schmid, Schmid Mast, 2010), этапы переработки информации (Люсин, Кожухова, 2016).

Возможно, полученные данные могут объясняться особенностями применения процедуры индукции эмоций в данной работе. Хотя были получены «нужные» сдвиги в оценках по шкалам позитивного и негативного аффекта, нужно отметить, что особенности индукции находятся в сильной зависимости от выборки участников исследования. В данном случае ее составили молодые люди - студенты, преимущественно женского пола, у которых исходные оценки позитивного аффекта уже были достаточно высоки. Следовательно, даже если эмоциональное состояние испытуемых стало еще более «радостным», то это привело к небольшому сдвигу в оценках позитивного аффекта во втором замере по сравнению с первым. Вследствие этого эффект конгруэнтности не получил сильной выраженности. Также нужно отметить, что шкала позитивного аффекта методики ШПАНА объединяет положительную валентность и эмоциональное возбуждение (arousal). В связи с этим процедура индукции положительного эмоционального состояния, которая применялась в исследовании, вероятнее всего, приводила как к смещению в оценках валентности состояния, так и к повышению уровня эмоционального воз- 
буждения (arousal). Поэтому остается открытым вопрос, объясняется ли полученный эффект настроения его валентностью или общим повышением активации.

В целом соотнесение результатов разных исследований, посвященных изучению эффекта конгруэнтности в распознавании эмоций, представляется трудным, так как в работах анализируются разные показатели распознавания (преимущественно точность), применяются разные способы индукции эмоций и проверки их эффективности. Проведенное исследование вносит вклад в изучение эффекта конгруэнтности в связи с анализом распознавания различных категорий эмоций. Неожиданный результат о замедлении распознавания радости по выражению лица требует дальнейшей эмпирической проверки и теоретического анализа других возможных факторов.

\section{Литература}

Кожухова Ю.А. (2016). Переработка аффективно окрашенной информации (на примере антисаккадной задачи). В кн. Седьмая международная конференция по когнитивной науке: Тезисы докладов (с. 323-325). Светлогорск.

Осин, Е. Н. (2012). Измерение позитивных и негативных эмоций: разработка русскоязычного аналога методики PANAS. Психология. Журнал Высшей школы экономики, 9(4), 91-110.

Осин, Е. Н., Леонтьев, Д. А. (2008). Апробация русскоязычных версий двух шкал экспрессоценки субъективного благополучия. В кн. Материалы ІІІ Всероссийского социологического конгресса. М.: Институт социологии РАН/Российское общество социологов. Режим доступа: http://publications.hse.ru/en/chapters/78753840.

Bower, G. H. (1981). Mood and memory. American Psychologist, 36, 129-148.

Calvo, M. G., Nummenmaa, L., \& Avero, P. (2010). Recognition advantage of happy faces in extrafoveal vision: Featural and affective processing. Visual Cognition, 18(9), 1274-1297.

Chentsova Dutton, Y. E., Parrott, G., \& Lyusin, D. (2013). Culture and perceived functions of sadness. Paper presented at the 14th Annual Meeting of the Society for Personality and Social Psychology, New Orleans, Louisiana, USA.

Chepenik, L. G., Cornew, L. A., \& Farah, M. J. (2007). The influence of sad mood on cognition. Emotion, 7, 802-811.

Edwards, M. S. (2014). Attentional biases in processing emotional facial expressions: Effects of state anxiety, trait anxiety and awareness. International Journal of Psychological Studies, 6(4), 41-55.

Quarto, T., Blasi, G., Pallesen, K. J., Bertolino, A., \& Brattico, E. (2014). Implicit processing of visual emotions is affected by sound-induced affective states and individual affective traits. PLoS ONE, 9(7), e103278. doi:10.1371/journal.pone.0103278

Rafienia, P., Azadfallah, P., Fathi-Ashtiani, A., \& Rasoulzadeh-Tabatabaiei, K. (2008). The role of extraversion, neuroticism and positive and negative mood in emotional information processing. Personality and Individual Differences, 44, 392-402.

Rusting, C. L. (1998). Personality, mood, and cognitive processing of emotional information: three conceptual frameworks. Psychological Bulletin, 124, 165-196. 
Schmid, P. C., \& Schmid Mast, M. (2010). Mood effects on emotion recognition. Motivation and Emotion, 34, 288-292.

Tamir, M., Robinson, M. D., \& Clore, G. L. (2002). The epistemic benefits of trait-consistent mood states: An analysis of extraversion and mood. Journal of Personality and Social Psychology, 83(3), 663-677.

Van der Schalk, J., Hawk, S. T., Fischer, A. H., \& Doosje, B. J. (2011). Moving faces, looking places: The Amsterdam Dynamic Facial Expressions Set (ADFES). Emotion, 11, 907-920.

Westermann, R., Spies, K., Stahl, G., \& Hesse, F. W. (1996). Relative effectiveness and validity of mood induction procedures: A meta-analysis. European Journal of Social Psychology, 26, 557-580.

Овсянникова Виктория Владимировна - старший научный сотрудник, Национальный исследовательский университет «Высшая школа экономики», кандидат психологических наук.

Контакты: v.ovsyannikova@gmail.com

\title{
The Effect of Emotional State and Dispositional Joy on the Speed of Emotion Recognition from Facial Expression
}

\author{
V.V. Ovsyannikova ${ }^{a}$ \\ ${ }^{a}$ National Research University Higher School of Economics, 20 Myasnitskaya str., Moscow, 101000, \\ Russian Federation
}

\begin{abstract}
Previous works show that mood congruence effect or trait congruence effect can be achieved (Chepenik et al., 2007; Rusting, 1998). The present study explores the effect of emotional state and dispositional joy on effectiveness of emotion recognition from facial expression. The experimental study was conducted in two groups of subjects. The general sample consisted of 39 participants. Participants' emotional state was measured with the self-report questionnaire PANAS. The participants' current mood was manipulated with the emotion induction procedure, which involved screening video with "joyful" or "neutral" emotional coloring. To measure the speed of emotional information processing a computer technique was used, in which a participant performed the task on emotion recognition from facial expression. The hypothesis was tested whether there is an effect of congruency in positive information processing. It was supposed that positive emotional state and dispositional joy heighten the speed of positive information processing and don't influence processing of the stimuli with negative emotional coloring. Testing of the emotion induction procedure proved it to be partially successful. Congruency effect for dispositional joy was achieved: we found an interrelation of higher manifestation of this trait with higher speed in joy recognition from facial expressions. The influence of positive emotional state was manifested in lower speed in recognition of joy. In sum, the results show that the congruency effect is expressed differently for trait and emotional state. Overall, the results of the conducted study provide information on the mechanisms of emotion recognition.
\end{abstract}

Keywords: emotion recognition, emotional state, emotion induction, congruence. 


\section{References}

Bower, G. H. (1981). Mood and memory. American Psychologist, 36, 129-148.

Calvo, M. G., Nummenmaa, L., \& Avero, P. (2010). Recognition advantage of happy faces in extrafoveal vision: Featural and affective processing. Visual Cognition, 18(9), 1274-1297.

Chentsova Dutton, Y. E., Parrott, G., \& Lyusin, D. (2013). Culture and perceived functions of sadness. Paper presented at the 14th Annual Meeting of the Society for Personality and Social Psychology, New Orleans, Louisiana, USA.

Chepenik, L. G., Cornew, L. A., \& Farah, M. J. (2007). The influence of sad mood on cognition. Emotion, 7, 802-811.

Edwards, M. S. (2014). Attentional biases in processing emotional facial expressions: Effects of state anxiety, trait anxiety and awareness. International Journal of Psychological Studies, 6(4), 41-55.

Kozhukhova, Yu. A., \& Lyusin, D. V. (2016). Rol' emotsional'nykh chert nablyudatelya pri vospriyatii emotsional'nykh lits na rannem etape pererabotki informatsii [The role of observer's emotional traits in perception of emotional faces at the early stage of information processing]. Manuscript in preparation.

Osin, E. N. (2012). Measuring Positive and Negative Affect: Development of a Russian-language Analogue of PANAS. Psychology. Journal of Higher School of Economics, 9(4), 91-110. (in Russian)

Osin, E. N., \& Leontiev, D. A. (2008). Aprobatsiya russkoyazychnykh versii dvukh shkal ekspressotsenki sub"ektivnogo blagopoluchiya [Approbation of the Russian versions of two scales for express-evaluation of subjective well-being]. In Materialy III Vserossiiskogo Sotsiologicheskogo Kongressa [Proceedings of the III All-Russian Sociological Congress]. Moscow: Institute of Sociology of the Russian Academy of Sciences / Russian Society of Sociologists. Retrieved from http://publications.hse.ru/en/chapters/78753840

Quarto, T., Blasi, G., Pallesen, K. J., Bertolino, A., \& Brattico, E. (2014). Implicit processing of visual emotions is affected by sound-induced affective states and individual affective traits. PLoS ONE, 9(7), e103278. doi:10.1371/journal.pone.0103278

Rafienia, P., Azadfallah, P., Fathi-Ashtiani, A., \& Rasoulzadeh-Tabatabaiei, K. (2008). The role of extraversion, neuroticism and positive and negative mood in emotional information processing. Personality and Individual Differences, 44, 392-402.

Rusting, C. L. (1998). Personality, mood, and cognitive processing of emotional information: three conceptual frameworks. Psychological Bulletin, 124, 165-196.

Schmid, P. C., \& Schmid Mast, M. (2010). Mood effects on emotion recognition. Motivation and Emotion, 34, 288-292.

Tamir, M., Robinson, M. D., \& Clore, G. L. (2002). The epistemic benefits of trait-consistent mood states: An analysis of extraversion and mood. Journal of Personality and Social Psychology, 83(3), 663-677.

Van der Schalk, J., Hawk, S. T., Fischer, A. H., \& Doosje, B. J. (2011). Moving faces, looking places: The Amsterdam Dynamic Facial Expressions Set (ADFES). Emotion, 11, 907-920.

Westermann, R., Spies, K., Stahl, G., \& Hesse, F. W. (1996). Relative effectiveness and validity of mood induction procedures: A meta-analysis. European Journal of Social Psychology, 26, 557-580.

Victoria V. Ovsyannikova - senior researcher, National Research University Higher School of Economics, Ph.D.

E-mail: v.ovsyannikova@gmail.com 